\title{
Presencia de Listeria monocytogenes en alimentos listos para el consumo, procedentes de plazas de mercado y delicatessen de supermercados de cadena, Bogotá, D.C, 2002-2008
}

\author{
Ana Isabel Muñoz, Mercedes Vargas, Ligia Otero, Graciela Díaz, Viviana Guzmán \\ Laboratorio de Microbiología de Alimentos, Subdirección de Alimentos y Bebidas Alcohólicas, \\ Instituto Nacional de Vigilancia de Medicamentos y Alimentos, Bogotá, D.C., Colombia
}

Introducción. El aumento de las enfermedades transmitidas por alimentos se ha constituido en un problema de salud pública. La listeriosis, cuyo agente etiológico es Listeria monocytogenes, es considerada de gran relevancia entre las ellas y los alimentos listos para el consumo de ventas al por menor son de gran riesgo.

Objetivo. Identificar la presencia de L. monocytogenes en alimentos listos para el consumo, procedentes de plazas de mercado y delicatessen de Bogotá.

Materiales y métodos. Se llevó a cabo un estudio descriptivo transversal con componente analítico, en el cual se analizaron 600 alimentos, 300 de delicatessen y 300 de plazas de mercado. Se utilizaron metodologías de referencia de presencia o ausencia de L. monocytogenes en $25 \mathrm{~g}$ o mililitros de alimento.

Resultados. De las 600 muestras analizadas, 68 fueron positivas para L. monocytogenes (11,3\%), $26(38,25 \%)$ procedieron de delicatessen, $42(61,76 \%)$ de plazas de mercado. El serotipo aislado con mayor frecuencia fue $4 \mathrm{~b}$ en 53 (78 \%) aislamientos. Los quesos frescos y los quesos madurados mostraron mayor contaminación de $L$. monocytogenes que el resto de alimentos del estudio $(p=0,0009 / 0,0003)$.

Conclusión. Los resultados indican que estos alimentos son vehículos de transmisión del microorganismo, convirtiéndolos en potenciales alimentos de alto riesgo; deben ser vigilados y controlados por la autoridad competente. Se requieren programas para implementar la normativa sobre vigilancia, reducción y control de este microorganismo con miras hacia la prevención de las enfermedades transmitidas por alimentos.

Palabras clave: Listeria monocytogenes, enfermedades transmitidas por alimentos, listeriosis, salud pública.

Presence of Listeria monocytogenes in ready-to-eat foods available in open markets, delicatessens and supermarkets, Bogotá, 2002-2008

Introduction. The increase in diseases transmitted by foods has become a public health problem. Listeria monocytogenes, the etiological agent of listeriosis or typical food poisoning, is considered to be among the most important food borne illnesses. High risk foods for L.monocytogenes have been recognized as foods already prepared for consumption and made available in retail outlets.

Objective. The presence of $L$. monocytogenes was identified in prepared foods found in open markets and delicatessens in the city of Bogotá.

Material and methods. A transverse descriptive study over a 7-year period, with an analytic component, in which 600 foods were analyzed--300 from delicatessens and 300 from open market places. Reference methods were used to determine the presence or absence of $L$. monocytogenes in 25 grams or milliliters a food sample.

Results. Of the 600 samples, 68 were positive for L. monocytogenes (11.3\%). Twenty-six (38.2\%) were taken from delicatessens and $42(61.8 \%)$ from open market places. The serotype most frequently isolated was $4 \mathrm{~b}$, with $53(78.0 \%)$ isolations. Fresh cheeses and matured cheeses showed greater contamination with $L$. monocytogenes than the other foods ( $p<0.001$ in both cases).

Conclusions. The results indicate that prepared foods are vehicles for the transmission of the Listeria bacterium. These potentially high risk foods are candidates for control and monitoring by public health authorities. Programs must be instituted to implement surveillance norms that lead to the reduction and control of this microorganism and geared toward the prevention of food borne diseases.

Key words: Listeria monocytogenes, foodborne diseases, listeriosis, public health. 
Las enfermedades transmitidas por alimentos constituyen un problema de salud pública. Es así como la Organización de las Naciones Unidas para la Agricultura y Alimentación (Food and Agriculture Organization, FAO) y la Organización Mundial de la Salud (OMS) están preocupadas por el aumento en la incidencia de estas enfermedades en las últimas décadas. Se desconoce si es el resultado de una mejor vigilancia y utilización de metodologías con una mejor caracterización de los microorganismos (1).

El aumento de las enfermedades transmitidas por alimentos podría ser real, debido a las tendencias observadas en hábitos alimentarios, como el consumo de cierto tipo de alimentos $(2,3)$, el aumento de las poblaciones en riesgo (2-5), como es el caso de personas inmunodeprimidas $(2,4-8)$, o la globalización de los mercados que hacen posible que un alimento contaminado con un patógeno produzca una de estas enfermedades en un país diferente al de origen, todo esto unido a una mejor vigilancia epidemiológica y una mayor tecnificación en los métodos para aislar e identificar patógenos en los alimentos.

La listeriosis, cuyo agente etiológico es Listeria monocytogenes $(2,4,5,9-20)$, se sitúa entre las enfermedades transmitidas por alimentos de mayor relevancia en la salud pública, debido al impacto social y económico que tiene por la gravedad de su cuadro clínico $(1,6,19,21,22)$.

En los países industrializados, como Estados Unidos, en donde la listeriosis es de notificación obligatoria en todos los estados desde el 2001 (23), se reportan 2.500 casos anuales de listeriosis ocasionados por alimentos y muere una de cada cinco personas afectadas (5,6,10,23-25).

Los Centers for Disease Control and Prevention (CDC) reportan 20 muertes por cada 100 casos de listeriosis por año (7) y consideran esta enfermedad como un importante problema de salud pública $(6,7,19)$. Los países pertenecientes a la Unión Europea reportan una incidencia de 2 a 10 casos de listeriosis por millón de personas (13).

En Colombia, los reportes de listeriosis son muy pobres. Se notificaron al Sistema Nacional de Vigilancia en Salud Pública (Sivigila) las

Correspondencia:

Ana Isabel Muñoz, Avenida calle 26 № 51-20, Bogotá, D.C. Colombia.

Teléfono: (571) 220 7700, extensión 1221; fax: (571) 2207700 , extensión 1221

amunozc@invima.gov.co y anisabel2001@yahoo.com

Recibido: 21/10/10; aceptado:28/06/11 enfermedades transmitidas por alimentos ocasionadas hasta la semana epidemiológica número 16 de 2010, el cual informó cuatro brotes de listeriosis (26). Aunque L. monocytogenes no es un microorganismo de notificación obligatoria, podría serlo en un futuro si se fortalece la vigilancia de dichas enfermedades.

Listeria monocytogenes es un bacilo Gram positivo, $(2,10,12)$ que crece a temperaturas de 1 a $45^{\circ} \mathrm{C}$, con temperatura óptima de 30 a $37^{\circ} \mathrm{C}(2,12,27,28)$; tiene la habilidad de soportar temperaturas de refrigeración $(2,9,12,29-31)$ y es capaz de desarrollarse a pH de 4,4 a 9,6 (2,28,32-34). Asimismo, crece en concentraciones altas de cloruro de sodio $(15 \%)(2,27,33,34)$.

Es un microorganismo ambiental que tiene la facultad de adherirse a las superficies, formando biopelículas $(20,30,32,35)$ para protegerse de la acción de los tratamientos antimicrobianos $(20,30,35)$. En el ambiente de fábrica de producción de alimentos, tiene la oportunidad de contaminar en sus diferentes etapas, siendo esta vía la más frecuente para llegar al ser humano $(2,5,12,30)$.

Listeria monocytogenes es un patógeno, facultativo intracelular y oportunista $(1,2,4,5,9,10,24,32,36)$. Sus manifestaciones clínicas se pueden agrupar en dos categorías: listeriosis invasiva y listeriosis no invasiva $(1,16,17,28)$. La listeriosis invasiva $(2,24,28,37)$ se produce cuando la infección ocasionada por las células bacterianas atraviesa la barrera intestinal y alcanza sistemas y órganos que son blanco de infección, como son el sistema nervioso central y el útero en la mujer embarazada $(1,2,11,13,37)$, ocasionando meningitis $(2,9,11$, $12,15,19,21,24,31,36)$, bacteriemias $(2,3,9,12,19,31)$, septicemias $(2,9,11,13,15,19,21,24,31,36)$, meningoencefalitis $(2,9,21,24,31)$, absceso cerebral o espinal, listeriosis pontobulbar y romboencefalitis $(2,3,13,21,30,38,39)$.

En la mujer embarazada se disemina por vía sanguínea y atraviesa la barrera transplacentaria $(2,11,40)$, lo que conlleva a una infección en el feto 0 en el recién nacido que da origen a abortos espontáneos $(2,9,11,15,19,21,31,36)$, muerte fetal, septicemia neonatal grave, muerte neonatal, meningitis infantil y granulomatosis $(2,4,12,13,15,19,41,42)$.

La listeriosis no invasiva se manifiesta por síntomas de gastroenteritis $(1,5,28,43)$, como diarrea, fiebre, cefalea y mialgias, y su periodo de incubación es corto $(1,4,13,15,19,21,28,43-45)$. 
La listeriosis invasiva afecta a los grupos de población más vulnerables como: mujeres embarazadas, niños en periodo neonatal, ancianos y personas con depresióndelsistemainmunológicoporenfermedades como el síndrome de inmunodeficiencia adquirida (sida), por medicamentos como la cortisona o por hábitos como el alcoholismo (1-5,8,10,13,1720,46).

A pesar de que la listeriosis es de rara presentación, comparada con otras enfermedades transmitidas por alimentos $(2,4,24,30)$, su mortalidad es alta, con un promedio de 20 a $30 \%(1,2,4,5,8,9,13,14,1$ $7,20,21,24,30,47)$.

Existen 13 serotipos:1/2a,1/2b,1/2c,3a,3b,3c,4a, $4 a b, 4 b, 4 c, 4 d, 4 e, 7 \quad(2,10,30,32,36,48-50)$, de los cuales se han encontrado implicados con mayor frecuencia en los brotes de listeriosis humanas los siguientes: 4b, 1/2a y $1 / 2 b(2,4,9,10,20,24,30,32,48-$ $51), 1 / 2 \mathrm{c}(2,30,34,36)$, mientras que, en ambientes de fábrica y en alimentos, se han aislado con más frecuencia los serotipos 1/a y 1/2b $(10,20,30,36,49)$. Para efectos de la salud pública, todos los serotipos de L. monocytogenes son patógenos $(4,21)$.

En brotes y casos de listeriosis siempre se ha encontrado una fuente alimentaria $(2,4,30-32,52)$ y los alimentos implicados han sido: quesos $(2,52-$ $54)$, leches $(2,43,55)$, patés $(2,56,57)$, perros calientes (58), ensalada de repollo $(2,59)$, pollo $(2,60)$, salami $(2,52,61)$, hortalizas $(2,61)$, carne de pavo (58), cárnicos (32), cárnicos listos para el consumo (52), embutidos (52) y lengua de cerdo (62).

Los alimentos considerados de alto riesgo para contraer listeriosis son los listos para el consumo $(1,5,9-11,16,17,28,30-32,34,47,50,63-66)$. Ésta es una categoría amplia y heterogénea de alimentos (incluye bebidas) que no van a tener ningún proceso de cocción antes de su consumo, o que aparentemente son aptos para el consumo sin cocción $(5,16)$, y varían de unos países a otros en relación con los hábitos alimentarios, la disponibilidad de la cadena de frío y la temperatura máxima en el punto de venta $(1,5)$.

En la guía para el cumplimiento de políticas sobre la vigilancia de L. monocytogenes en Estados Unidos, las agencias responsables son la Food and Drug Administration (FDA), el Food Safety and Inspection Service (FSIS) del United States Department of Agriculture (USDA) y los CDC. Dichas agencias hacen referencia al estudio realizado en el 2003 sobre aseguramiento de riesgos relacionados con la listeriosis y clasifican los alimentos listos para el consumo en dos categorías $(5,16)$.

La primera categoría corresponde a alimentos listos para el consumo que tienen un alto riesgo de asociarse con listeriosis debido a que facilitan el crecimiento de $L$. monocytogenes: leches, derivados lácteos con gran contenido de grasas, como quesos frescos, mantequillas, cremas, crustáceos cocidos, productos de mar crudos y ahumados, vegetales, frutas ácidas y otros $(5,16)$. EI FSIS agrupa en cárnicos y aves, los alimentos como salchichas, ensaladas, patés, perros calientes, salamis, peperonis, jamones cocidos, jamones curados, prosciutto, rollos de carne, carnes en tajadas, carnes para untar, carnes cortadas en porciones, carnes molidas, hamburguesas, presas de pollo, nuggets de pollo, tajadas de jamón de carne de pollo y de pavo, pizzas, rollos de huevo, sándwiches, ensaladas y otros $(64,67)$.

En la segunda categoría, encontramos los alimentos listos para el consumo que tienen poco riesgo de asociarse con listeriosis, debido a que no facilitan el crecimiento de L. monocytogenes; entre ellos se encuentran los alimentos que tienen factores intrínsecos, como pH de 4,4 o menos, actividad de agua de 0,92 o menor, humedad relativa menor de $39 \%$, y factores extrínsecos, como los ambientales relacionados con temperaturas de conservación; alimentos como helados o productos de congelación; alimentos con proceso de marinado, encurtidos o deshidratados; alimentos con conservantes como ácido cítrico, benzoatos; vegetales como la zanahoria; leches fermentadas como el yogur; quesos duros; y ensaladas procesadas con ácido sórbico o benzoico con pH de 4,4 o menos $(5,16)$.

Con la clasificación alimentaria expuesta anteriormente, el criterio establecido en los Estados Unidos para aceptar como inocuos los alimentos listos para el consumo en los que puede crecer L. monocytogenes, es de cero tolerancia $(10,16,47,48,65,68,69)$. Europa acepta niveles hasta de 100 unidades formadoras de colonias (UFC) para algunos alimentos que no estén destinados a poblaciones vulnerables (47).

El objetivo de este estudio fue estimar la presencia de $L$. monocytogenes en alimentos listos para el consumo en puntos de venta al por menor, procedentes de plazas de mercado y delicatessen de supermercados de cadena, en la ciudad de Bogotá, los cuales fueron clasificados en 18 grupos de alimentos. Se consideraron favorables para el crecimiento de este microorganismo características 
como matriz del alimento, $\mathrm{pH}$, actividad de agua, humedad relativa, conservación y temperatura en el momento de la toma de la muestra.

\section{Materiales y métodos}

Se hizo un estudio descriptivo y transversal durante los años 2002 a 2008, para determinar la presencia de $L$. monocytogenes en alimentos listos para el consumo, de ventas al por menor, procedentes de plazas de mercado y delicatessen que existen en Bogotá. No fue posible establecer el número total de este tipo de alimentos que se vende al por menor en las plazas de mercado y en los delicatessen; la población se asume muy grande para efectos del tamaño muestral.

Se utilizó el programa Epilnfo, versión 6.04, definiendo para su estimación una probabilidad de $50 \%$ (por ser desconocida), un error esperado de $4 \%$ y una significancia del $95 \%$. Se estableció un tamaño de 600 muestras; 300 muestras fueron tomadas en los delicatessen de todos los supermercados de cadena registrados en Bogotá $y$, las otras 300 muestras, en todas las plazas de mercado registradas en Bogotá.

Todos los establecimientos fueron codificados; a los delicatessen se les asignó una codificación de 01 a 12 y, a las plazas de mercado, de 20 a 26. El número de muestras tomadas en cada establecimiento se calculó de manera ponderada (cuadro 1). En el primer caso, se tuvo en cuenta el número de locales con delicatessen pertenecientes al mismo establecimiento, utilizando un muestreo aleatorio simple.

Con las plazas de mercado, se tuvo en cuenta el número de locales de ventas al por menor de alimentos listos para el consumo. Los alimentos se clasificaron en los siguientes grupos: aves, bebidas, cárnicos ahumados, cárnicos cocidos, cárnicos madurados, comidas preparadas, comidas rápidas, crema de leche, empanadas y pasteles, encurtidos, ensaladas, jugos, leches fermentadas, productos de panadería, productos derivados de la pesca, postres, quesos frescos, quesos fundidos , quesos madurados y salsas.

La cantidad de muestra tomada por alimento fue de $500 \mathrm{~g}$ o mililitros, y la toma se hizo de manera aleatoria por las profesionales del Laboratorio de Microbiología de Alimentos del Invima en compañía del personal de Vigilancia de Alimentos de este Instituto. Las muestras se transportaron en bolsas estériles a temperaturas de refrigeración y se analizaron en el Laboratorio de Microbiología de Alimentos el mismo día de la toma de la muestra.

El muestreo se hizo durante los años 2002 a 2006, de la siguiente manera: en el 2002 se tomaron y analizaron 63 muestras; en el 2003, 204 muestras; en el 2004, 290 muestras; en el 2005, 10 muestras; en el 2006, 33 muestras.

Las serotipificaciones de los aislamientos encontrados se hicieron en los años 2007 y 2008. El control de calidad externo para las serotipificación de los aislamientos encontrados, estuvo a cargo del Laboratorio de Listeria del Instituto Pasteur de París y Centro Colaborativo de la OMS para la Listeriosis de Origen Alimentario, mediante la concordancia en los resultados.

Para el aislamiento y la identificación de $L$. monocytogenes, se usó la metodología recomendada en alimentos por la FDA (70), y la metodología adaptada de la FDA (2003) en derivados cárnicos, huevos y pollos (71), ambas validadas por el Laboratorio de Microbiología de Alimentos del Invima, metodologías de presencia o ausencia en $25 \mathrm{~g}$ o mililitros de alimento, debido a que nuestro país al igual que Estados Unidos $(5,10,16,47,48,65)$ como también por recomendaciones del Codex Alimentarius (72), no contempla ningún nivel de aceptabilidad para alimentos listos para el consumo que soportan promueven o facilitan el crecimiento de L. monocytogenes. Para la tipificación serológica

Cuadro 1. Número de muestras tomadas por establecimiento

\begin{tabular}{lrr}
\hline Establecimiento & $\mathbf{n}$ & $\%$ \\
\hline Código 01 & 19 & 3,2 \\
Código 02 & 31 & 5,7 \\
Código 03 & 41 & 7,0 \\
Código 04 & 40 & 7,0 \\
Código 05 & 24 & 4,0 \\
Código 06 & 5 & 0,8 \\
Código 07 & 11 & 1,8 \\
Código 08 & 37 & 6,2 \\
Código 09 & 32 & 5,3 \\
Código10 & 20 & 3,3 \\
Código 11 & 3,3 \\
Código 12 & 20 & 3,3 \\
Código 20 & 20 & 3,3 \\
Código 21 & 20 & 6,7 \\
Código 22 & 40 & 6,7 \\
Código 23 & 40 & 6,7 \\
Código 24 & 40 & 6,7 \\
Código 25 & 40 & 6,7 \\
Código 26 & 40 & 13,3 \\
Total & 80 & 100 \\
\hline
\end{tabular}

Códigos delicatesen: 01-12; códigos plazas de mercado: 20-26. 
se utilizó la metodología de Seeliger y Höhne (73), desarrollada y enseñada por el Laboratorio de Listeria del Instituto Pasteur de París y verificada por el Laboratorio de Microbiología de Alimentos del Invima (74).

Para el análisis de resultados se utilizaron los programas Microsoft Office Excel® 97-2003 y el programa Epi-Info®, versión 3.5.1. Mediante análisis univariados y multivariados, se estimaron proporciones, medidas de tendencia central, dispersión y posición. Se calculó la razón de resultados positivos entre proporciones, dada por la procedencia y los diferentes grupos de alimentos, a fin de establecer una hipótesis de asociación; también, se aplicaron pruebas de significancia estadística.

\section{Resultados}

De las 600 muestras analizadas de alimentos listos para el consumo de ventas al por menor, 68 fueron positivas para detección de $L$. monocytogenes, lo que corresponde a $11,3 \%$. Todos los alimentos analizados fueron clasificados por grupos. Los alimentos que se muestrearon con mayor frecuencia fueron los quesos frescos, cárnicos cocidos y ensaladas (cuadro 2). De estas muestras para $L$. monocytogenes, se aislaron los siguientes serotipos: $1 / 2 \mathrm{a}$ en un aislamiento $(1,4$ $\%) ; 1 / 2 b$ en dos aislamientos (3\%); $4 b$ en 53 aislamientos (78\%); y 4d-4e en 12 aislamientos (17,6 \%) (figura1).

La distribución de frecuencias para cada serotipo, según el tipo de alimento se presenta en el cuadro 3.

Las muestras positivas para L. monocytogenes procedentes de los delicatessen fueron 26 (38,25 $\%$ y las procedentes de plazas de mercado fueron $42(61,76 \%)$. La proporción de resultados positivos en las plazas de mercado fue de $7 \%$ y, en los delicatessen, de 4,33 \%; la razón entre proporciones fue de 1,62 , con IC $95 \%(1,02-$ 2,56).

El serotipo aislado con mayor frecuencia, tanto en plazas de mercado como en delicatessen, fue el serotipo 4b. No se observaron diferencias en la distribución de los serotipos aislados entre plazas de mercado y delicatessen $(p=0,297)$ (cuadro 4 , figura 2). La distribución de los serotipos de $L$. monocytogenes aislados de acuerdo con el grupo de alimentos en plazas de mercado, se observa en la figura 3.

La distribución de los serotipos de L. monocytogenes aislados de acuerdo con el grupo de alimentos en los delicatessen, se observa en la figura 4.

Hay diferencias estadísticas en los resultados positivos para $L$. monocytogenes en quesos frescos y quesos madurados $(p=0,0009 / 0,0003)$

Cuadro 2. Distribución de resultados positivos para Listeria monocytogenes en las muestras según grupo de alimentos, Bogotá, 2002-2008

\begin{tabular}{|c|c|c|c|c|c|c|}
\hline Grupo de alimentos & $\begin{array}{l}\text { Positivas } \\
\text { (n) }\end{array}$ & $\%$ & $\begin{array}{c}\text { Negativas } \\
\text { (n) }\end{array}$ & $\%$ & $\begin{array}{c}\text { Total } \\
\text { (n) }\end{array}$ & $\%$ \\
\hline Aves & 0 & 0 & 14 & 2,3 & 14 & 2,3 \\
\hline Bebidas & 0 & 0 & 3 & 0,5 & 3 & 0,5 \\
\hline Cárnicos ahumados & 1 & 0,2 & 1 & 0,2 & 2 & 0,3 \\
\hline Cárnicos cocidos & 8 & 1,3 & 105 & 17,5 & 113 & 18,8 \\
\hline Cárnicos madurados & 0 & 0 & 5 & 0,8 & 5 & 0,8 \\
\hline Comidas preparadas & 5 & 0,8 & 54 & 9 & 59 & 9,8 \\
\hline Comidas rápidas & 3 & 0,5 & 37 & 6,2 & 40 & 6,7 \\
\hline Crema de leche & 1 & 0,2 & 8 & 1,3 & 9 & 1,5 \\
\hline Empanadas y pasteles & 1 & 0,2 & 31 & 5,2 & 32 & 5,3 \\
\hline Encurtidos & 0 & 0 & 1 & 0,2 & 1 & 0,2 \\
\hline Ensaladas & 12 & 2 & 58 & 9,7 & 70 & 11,7 \\
\hline Jugos & 0 & 0 & 8 & 1,3 & 8 & 1,3 \\
\hline Leches fermentadas & 0 & 0 & 1 & 1,2 & 1 & 1,2 \\
\hline Productos de panadería & 2 & 0,3 & 47 & 7,8 & 49 & 8,1 \\
\hline Productos derivados de la pesca & 4 & 0,7 & 22 & 3,7 & 26 & 4,3 \\
\hline Postres & 2 & 0,3 & 32 & 5,3 & 34 & 5,7 \\
\hline Quesos frescos & 23 & 3,8 & 91 & 15,2 & 114 & 19 \\
\hline Quesos fundidos & 0 & 0 & 3 & 0,5 & 3 & 0,5 \\
\hline Quesos madurados & 6 & 1 & 9 & 1,5 & 15 & 2,5 \\
\hline Salsas & 0 & 0 & 2 & 0,3 & 2 & 0,3 \\
\hline Total & 68 & 11,3 & 532 & 88,7 & 600 & 100 \\
\hline
\end{tabular}




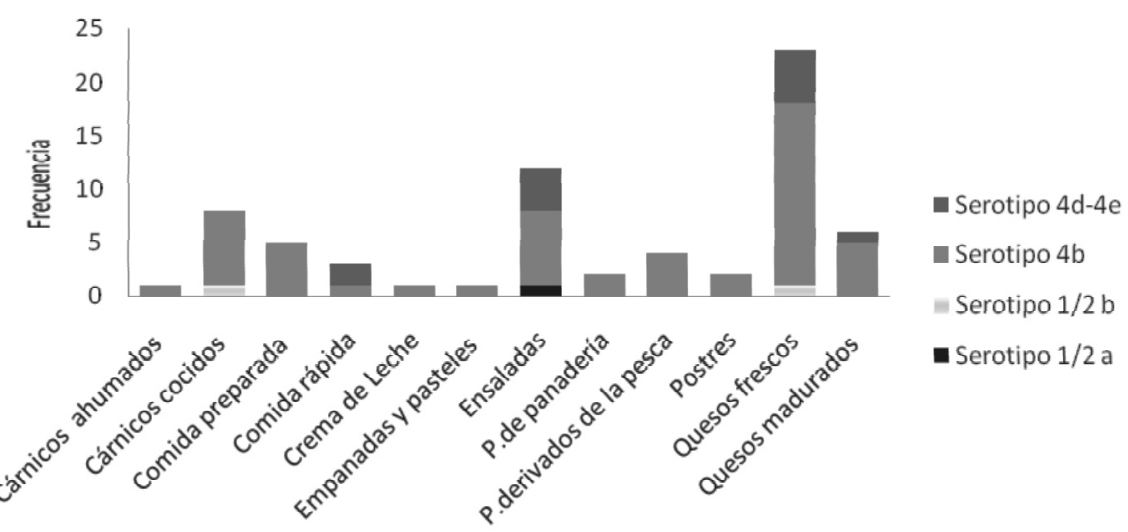

Figura 1. Serotipos de Listeria monocytogenes aislados en alimentos listos para el consumo procedentes de plazas de mercado y delicatessen por grupos de alimentos. Bogotá, 2002-2008.

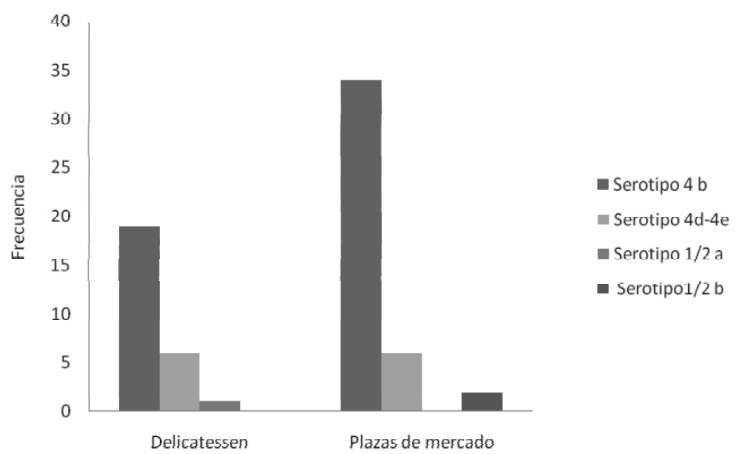

Figura 2. Serotipos de Listeria monocytogenes en alimentos listos para el consumo, según sitio de toma de muestra.

con referencia al resto de grupos de alimentos considerados en la muestra (cuadro 5).

Se encontraron otras especies no patógenas del género Listeria. Del total de muestras analizadas, $41(6,83 \%)$ fueron positivas para otras especies de Listeria, 11 (1,83\%) pertenecientes a muestras tomadas en delicatessen y $30(5 \%)$ procedentes de plazas de mercado. De las 11 muestras positivas tomadas en los delicatessen, se encontraron: 5 $(45,4 \%)$ con Listeria innocua de serotipo $6 \mathrm{a}, 2$ $(18,2 \%)$ con $L$. innocua de serotipo 6 b y $4(36,4$ $\%$ ) con Listeria seeligeri de serotipo 1/2b. De las 30 muestras positivas para otras especies de Listeria procedentes de las plazas de mercado, se encontraron $19(63,3 \%)$ con L. innocua $6 a, 10$ $(33,3 \%)$ con $L$. innocua de serotipo 6 b y 1 (3,3\%) con $L$. seeligeri de serotipo $1 / 2 b$.

\section{Discusión}

Este estudio identifica el riesgo que podría constituir la venta al por menor de alimentos listos para el consumo contaminados con L. monocytogenes, en las plazas de mercado y en los delicatessen.
Dada la proporción de resultados positivos para L. monocytogenes en el presente estudio, se puede afirmar que el consumo indiscriminado en Colombia de estos alimentos podría ser un factor de riesgo mayor que en otros países, en los cuales desde hace algunas décadas se ha implementado la vigilancia para este patógeno.

Se encontró, en general, una presencia de L. monocytogenes en alimentos listos para el consumo de $11,33 \%$, muy alta, si la comparamos con el porcentaje de positivos $(0,43 \%)$ reportado por FSIS en los alimentos listos para el consumo que son competencia de su vigilancia (67).

La presencia de $L$. monocytogenes fue mayor en las muestras de alimentos listos para consumir tomadas en las plazas de mercado que en las muestras procedentes de los delicatessen; el riesgo de consumir alimentos contaminados con este microorganismo es 1,62 veces mayor si son adquiridos en plazas de mercado.

El serotipo aislado con mayor frecuencia, tanto en alimentos listos para el consumo de ventas al por menor procedentes de plazas de mercado, como los procedentes de delicatessen, fue el $4 \mathrm{~b}$, serotipo muy virulento y causante de la mayoría de casos y brotes de listeriosis en la humanidad, y el hallazgo de tan alta proporción de este serotipo incrementa el riesgo de adquirir listeriosis por el consumo de este tipo de alimentos; además, Colombia, como país en desarrollo, cuenta con un porcentaje de necesidades básicas insatisfechas de 27,78 \% (75), tan alto que afecta la calidad de vida de la población haciéndola más vulnerable de sufrir de listeriosis.

Este estudio demuestra que los quesos frescos y madurados son los grupos de alimentos más 
Cuadro 3. Distribución de los serotipos de Listeria monocytogenes aislados en los diferentes grupos de alimentos

\begin{tabular}{|c|c|c|c|c|c|c|c|c|c|c|c|c|}
\hline Tipo de alimento & Pos & $\%$ & $1 / 2 a$ & $\%$ & $1 / 2 b$ & $\%$ & $4 b$ & $\%$ & $4 d-4 e \%$ & Total & serotipos & $\%$ \\
\hline \multicolumn{13}{|c|}{ Grupo de quesos frescos } \\
\hline Cuajada & 6 & 26,1 & 0 & 0 & 0 & 0 & 5 & 21,7 & 1 & 4,3 & 6 & 26,1 \\
\hline Queso campesino & 8 & 34,8 & 0 & 0 & 0 & 0 & 6 & 26,1 & 2 & 8,7 & 8 & 34,8 \\
\hline Queso costeño & 2 & 8,7 & 0 & 0 & 1 & 4,3 & 1 & 4,3 & 0 & 0 & 2 & 8,7 \\
\hline Queso doble crema & 5 & 21,7 & 0 & 0 & 0 & 0 & 4 & 17,4 & 1 & 4,3 & 5 & 21,7 \\
\hline Queso tajado & 1 & 4,3 & 0 & 0 & 0 & 0 & 0 & 0 & 1 & 4,3 & 1 & 4,3 \\
\hline Suero & 1 & 4,3 & 0 & 0 & 0 & 0 & 1 & 4,3 & 0 & 0 & 1 & 4,3 \\
\hline Total muestras & 23 & 100 & 0 & 0 & 1 & 4,3 & 17 & 74 & 5 & 22 & 23 & 100 \\
\hline \multicolumn{13}{|l|}{ Grupo de ensaladas } \\
\hline De frutas & 4 & 33,3 & 1 & 8,3 & 0 & 0 & 2 & 16,7 & 1 & 8,3 & 4 & 33,3 \\
\hline De huevo & 1 & 8,3 & 0 & 0 & 0 & 0 & 1 & 8,3 & 0 & 0 & 1 & 8,3 \\
\hline De papa & 2 & 16,7 & 0 & 0 & 0 & 0 & 1 & 8,3 & 1 & 8,3 & 2 & 16,7 \\
\hline De verduras & 5 & 41,7 & 0 & 0 & 0 & 0 & 3 & 25 & 2 & 16,7 & 5 & 41,7 \\
\hline Total de muestras & 12 & 100 & 1 & 8,3 & 0 & 0 & 7 & 58,3 & 4 & 33,3 & 12 & 100 \\
\hline \multicolumn{13}{|c|}{ Grupo de cárnicos cocidos } \\
\hline Chicharrón de cerdo & 1 & 12,5 & 0 & 0 & 0 & 0 & 1 & 12,5 & 0 & 0 & 1 & 12,5 \\
\hline Galantina de pavo & 1 & 12,5 & 0 & 0 & 0 & 0 & 1 & 12,5 & 0 & 0 & 1 & 12,5 \\
\hline Jamón de cerdo & 3 & 37,5 & 0 & 0 & 0 & 0 & 3 & 37,5 & 0 & 0 & 3 & 37,5 \\
\hline Queso de cabeza & 3 & 37,5 & 0 & 0 & 1 & 12,5 & 2 & 25 & 0 & 0 & 3 & 37,5 \\
\hline Total muestras & 8 & 100 & 0 & 0 & 1 & 12,5 & 7 & 87,5 & 0 & 0 & 8 & 100 \\
\hline \multicolumn{13}{|c|}{ Grupo de quesos madurados } \\
\hline Tilsit & 1 & 16,6 & 0 & 0 & 0 & 0 & 1 & 16,6 & 0 & 0 & 1 & 16 \\
\hline Madurado & 1 & 16,6 & 0 & 0 & 0 & 0 & 1 & 16,6 & 0 & 0 & 1 & 16,6 \\
\hline Paipa & 4 & 67 & 0 & 0 & 0 & 0 & 3 & 66,7 & 1 & 16,6 & 4 & 66 \\
\hline Total & 6 & 100 & 0 & 0 & 0 & 0 & 5 & 83,3 & 1 & 16,6 & 6 & 10 \\
\hline \multicolumn{13}{|c|}{ Grupo de comidas preparadas } \\
\hline Arroz con pollo & 2 & 40 & 0 & 0 & 0 & 0 & 2 & 40 & 0 & 0 & 2 & 40 \\
\hline Espaguetis con pollo & 1 & 20 & 0 & 0 & 0 & 0 & 1 & 20 & 0 & 0 & 1 & 20 \\
\hline Fritanga & 1 & 20 & 0 & 0 & 0 & 0 & 1 & 20 & 0 & 0 & 1 & 20 \\
\hline Picada & 1 & 20 & 0 & 0 & 0 & 0 & 1 & 20 & 0 & 0 & 1 & 20 \\
\hline Total & 5 & 100 & 0 & 0 & 0 & 0 & 5 & 100 & 0 & 0 & 5 & 100 \\
\hline \multicolumn{13}{|c|}{ Grupo de derivados de la pesca } \\
\hline Ceviche & 1 & 25 & 0 & 0 & 0 & 0 & 1 & 25 & 0 & 0 & 1 & 25 \\
\hline Camarón & 2 & 50 & 0 & 0 & 0 & 0 & 2 & 50 & 0 & 0 & 2 & 50 \\
\hline Ostras & 1 & 25 & 0 & 0 & 0 & 0 & 1 & 25 & 0 & 0 & 1 & 25 \\
\hline Total & 4 & 100 & 0 & 0 & 0 & 0 & 4 & 100 & 0 & 0 & 4 & 100 \\
\hline \multicolumn{13}{|c|}{ Grupo de comidas rápidas } \\
\hline Arepa de choclo & 1 & 33,3 & 0 & 0 & 0 & 0 & 1 & 33,3 & 0 & 0 & 1 & 33,3 \\
\hline Papa cocida & 1 & 33,3 & 0 & 0 & 0 & 0 & 0 & 0 & 1 & 33,3 & 1 & 33,3 \\
\hline Sándwich de pollo & 1 & 33,3 & 0 & 0 & 0 & 0 & 0 & 0 & 1 & 33,3 & 1 & 33,3 \\
\hline Total & 3 & 100 & 0 & 0 & 0 & 0 & 0 & 33,3 & 2 & 66,6 & 3 & 100 \\
\hline \multicolumn{13}{|l|}{ Productos de panadería } \\
\hline Torta de queso & 1 & 50 & 0 & 0 & 0 & 0 & 1 & 50 & 0 & 0 & 1 & 50 \\
\hline Torta de piña & 1 & 50 & 0 & 0 & 0 & 0 & 1 & 50 & 0 & 0 & 1 & 50 \\
\hline Total & 2 & 100 & 0 & 0 & 0 & 0 & 2 & 100 & 0 & 0 & 2 & 100 \\
\hline \multicolumn{13}{|l|}{ Grupo de postres } \\
\hline Flan de melocotón & 1 & 50 & 0 & 0 & 0 & 0 & 1 & 50 & 0 & 0 & 1 & 50 \\
\hline Postre de tres leches & 1 & 50 & 0 & 0 & 0 & 0 & 1 & 50 & 0 & 0 & 1 & 50 \\
\hline Total & 2 & 100 & 0 & 0 & 0 & 0 & 2 & 100 & 0 & 0 & 2 & 100 \\
\hline \multicolumn{13}{|c|}{$\begin{array}{l}\text { Otros grupos :crema de leche, } \\
\text { cárnicos ahumados, empanadas } \\
\text { y pasteles }\end{array}$} \\
\hline Crema de leche & 1 & 100 & 0 & 0 & 0 & 0 & 1 & 100 & 0 & 0 & 1 & 100 \\
\hline Chuleta ahumada & 1 & 100 & 0 & 0 & 0 & 0 & 1 & 100 & 0 & 0 & 1 & 100 \\
\hline Pastel de yuca & 1 & 100 & 0 & 0 & 0 & 0 & 1 & 100 & 0 & 0 & 1 & 100 \\
\hline
\end{tabular}

Pos: muestras positivas con serotipos de Listeria monocytogenes 1/2a, 1/2b, 4b o 4d-4e 
contaminados $(p=0,009 / 0003)$ debido, posiblemente, a deficiencias de higiene en la manipulación a lo largo de toda la cadena de producción, procedimiento, conservación y transporte, fraccionamiento y manipulación en la venta de estos productos y que, unido todo esto a la riqueza de sus componentes, al pH, y a su constante de humedad, le van a permitir su desarrollo.

De los 23 quesos frescos con presencia de $L$. monocytogenes y con serotipo 4 b en su mayoría, 22 de ellos provenían de plazas de mercado y fueron elaborados en forma artesanal y posiblemente con leche sin pasteurizar, como es la costumbre de fabricación de estos quesos.

A pesar de que no hubo diferencias significativas en la presencia de L. monocytogenes en ensaladas en relación con el resto de muestras, su frecuencia es relevante posiblemente por deficiencias higiénicas en el lavado de vegetales y frutas que proceden de cultivos que han sido fertilizados con materia orgánica humana o animal o regados con agua contaminada, malas prácticas de manufactura que permiten la contaminación cruzada a lo largo de la producción, conservación y fraccionamiento, y cuyas temperaturas de refrigeración permiten su crecimiento.
Dado los resultados de este estudio, se recomienda:

I. la implementación de buenas prácticas de manufactura (BPM) a lo largo de toda la cadena alimentaria;

II. la implementación de un buen control de calidad, como es el sistema de análisis de peligros y puntos críticos de control, y

III. la implementación de vigilancia ambiental de $L$. monocytogenes en las fábricas de alimentos, como lo hacen países industrializados.

En el marco del acuerdo de medidas sanitarias y fitosanitarias al cual hace referencia el CONPES 3375 (76), Colombia se encuentra interesada en reducir la frecuencia de las enfermedades transmitidas por alimentos, como la listeriosis, y adoptar medidas basadas en la información científica obtenida en evaluaciones de riesgo y de su impacto en la salud pública.

Se recomienda, a las autoridades sanitarias directamente relacionadas con la vigilancia de las enfermedades transmitidas por alimentos, diseñar programas que apoyen la normatividad sobre vigilancia, reducción y control de este

Cuadro 4. Distribución de los serotipos de Listeria monocytogenes según sitio de muestreo

\begin{tabular}{|c|c|c|c|c|c|c|c|c|c|c|}
\hline Sitio & Serotipo & $\%$ & Serotipo & $\%$ & Serotipo & $\%$ & Serotipo & $\%$ & Total & $\%$ \\
\hline Delicatessen & 1 & 3,8 & 0 & 0 & 19 & 73,1 & 6 & 23,1 & 26 & 38,2 \\
\hline Plazas de mercado & 0 & 0 & 2 & 4,8 & 34 & 81 & 6 & 14,3 & 42 & 61,8 \\
\hline Total & 1 & 1,5 & 2 & 2,9 & 53 & 77,9 & 12 & 17,6 & 68 & 100 \\
\hline
\end{tabular}

Cuadro 5. Resultados positivos de Listeria monocytogenes en grupos de alimentos con relación al total de la muestra

\begin{tabular}{lccccc}
\hline Grupo de alimentos & $\begin{array}{c}\text { Positivas } \\
\text { (n) }\end{array}$ & $\begin{array}{c}\text { Negativas } \\
\text { (n) }\end{array}$ & $\begin{array}{c}\text { Complemento del total } \\
\text { muestras positivas }\end{array}$ & $\begin{array}{c}\text { Complemento del total } \\
\text { muestras negativas }\end{array}$ & $\begin{array}{c}\text { p } \\
\text { Cárnicos ahumados }\end{array}$ \\
Cárnicos cocidos & 1 & 1 & 67 & 531 & 0,213 \\
Comidas preparadas & 8 & 105 & 60 & 427 & 0,113 \\
Comidas rápidas & 5 & 54 & 63 & 478 & 0,466 \\
Crema de leche & 3 & 37 & 65 & 524 & 0,3126 \\
Empanadas y pasteles & 1 & 8 & 67 & 501 & 0,728 \\
Ensaladas & 12 & 31 & 67 & 474 & 0,101 \\
Productos de panadería & 2 & 58 & 56 & 485 & 0,103 \\
Productos derivados & 4 & 47 & 66 & 510 & 0,094 \\
de la pesca & 22 & 64 & 500 & 0,338 \\
Postres & 2 & 32 & 66 & 441 & 0,301 \\
Quesos frescos & 23 & 91 & 45 & 623 & 0,0009 \\
Quesos madurados & 6 & 9 & 0 & 600 & 0,0003 \\
Otros grupos de alimentos & 0 & 37 & & & - \\
\hline
\end{tabular}




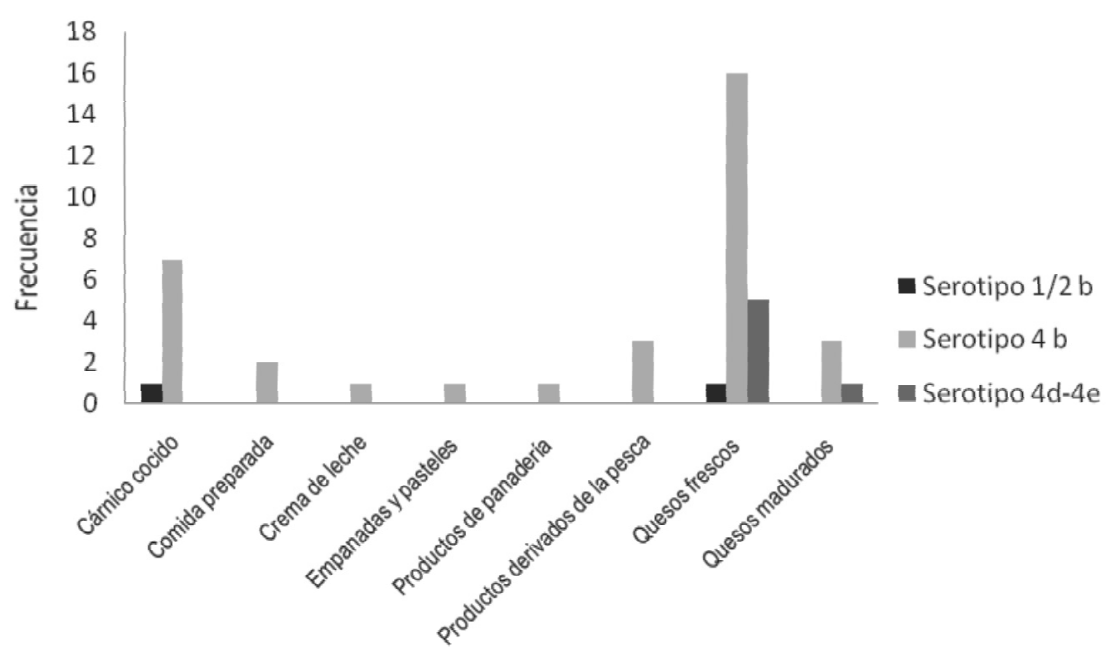

Figura 3. Distribución de serotipos de Listeria monocytogenes aislados de los grupos de alimentos procedentes de plazas de mercado.

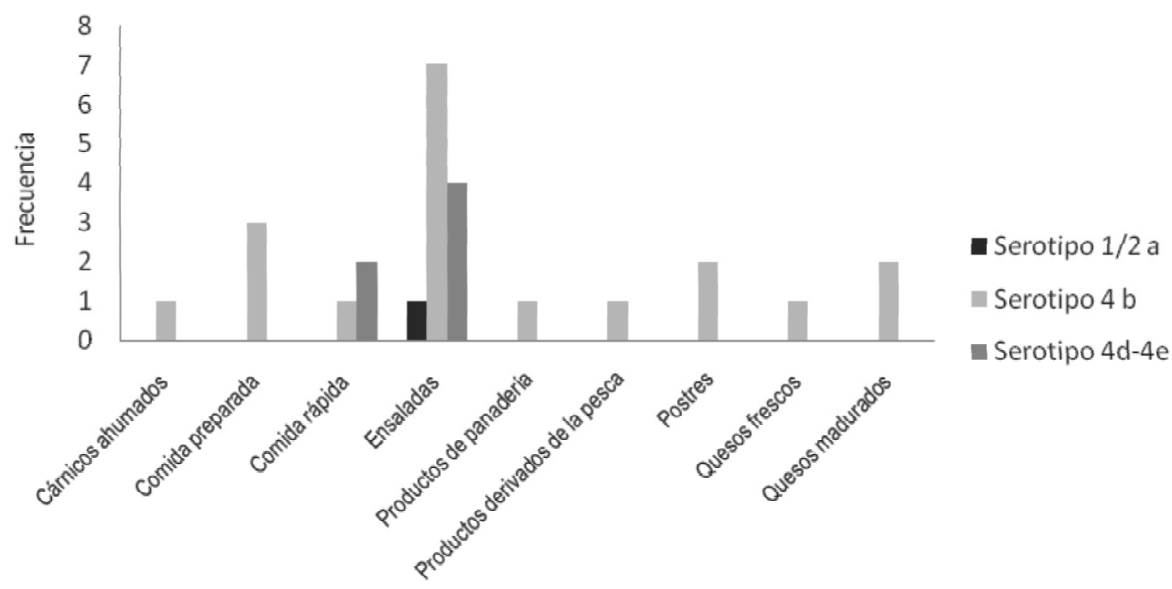

Figura 4. Distribución de serotipos de Listeria monocytogenes aislados de los grupos de alimentos procedentes de delicatessen.

microorganismo, en los cuales se enseñen medidas de prevención a las poblaciones en riesgo de adquirir listeriosis por el consumo de alimentos listos para el consumo, de ventas al por menor.

\section{Agradecimiento}

A Óscar Eduardo Pacheco, coordinador del Grupo de Epidemiología Aplicada (GEA) del Instituto Nacional de Salud, por la valiosa colaboración en el análisis e interpretación de resultados y la lectura crítica del presente artículo.

\section{Conflicto de intereses}

Los autores declaramos que durante la realización del trabajo no existió conflicto de intereses de ningún tipo.

\section{Financiación}

Este trabajo fue financiado por el Instituto Nacional de Vigilancia de Medicamentos y Alimentos (Invima).

\section{Referencias}

1. Organización de las Naciones Unidas para la Agricultura y la Alimentación. Organización Mundial de la Salud. Evaluación de riesgos de Listeria monocytogenes en alimentos listos para el consumo: resumen interpretativo. Serie de evaluación de riesgos microbiológicos No. 4. Roma: FAO/OMS; 2004. p. 53.

2. Muñoz Al, Díaz G. Listeriosis. Santafé de Bogotá: INVIMAINS; 1998. p. 64.

3. World Health Organization (WHO). Food borne listeriosis. WHO Working group. Bull World Health Organ. 1988;66:421-8. 
4. Rocourt J. Risk factors for listeriosis. Food Control. 1996;7:195-202.

5. Food and Drug Administration, Center for Food Safety and Applied Nutrition, USDA/Food Safety and Inspection Service, Centers for Disease Control and Prevention. Draf assessment of the relative risk to public health from foodborne Listeria monocytogenes among selected categories of ready- to-eat foods. Interpretative summary p.1-24, 2001. Fecha de consulta: 14 de febrero de 2002. Disponible en: http://www.foodsafety.gov/ dms/ Imrisk.html.

6. Division of Foodborne, Bacterial and Mycotic Diseases (DFBMD), Centers for Disease Control and Prevention. Listeriosis. Atlanta: CDC; 2008. p. 4. Fecha de consulta: 10 de mayo de 2009. Disponible en: http:// www.cdc.gov/ nczved/dfbmd/disease_listing/listeriosis_gi.html.

7. United States Department of Agriculture. Food Safety and Inspection Service. Production and inspection. FSIS rule designed to reduce Listeria monocytogenes in ready to eat food meat and poultry. USA: FSIS; 2009. p. 3. Fecha de consulta: 3 de mayo de 2009. Disponible en: http://www. fsis.usda.gov/factsheets/fsis_rule_designed_to_reduce_ listeria/index.asp.

8. Canadian Food Inspection Agency. Food safety facts on Listeria. Canada: CFIA; 2010 p. 4. Fecha de consulta: 10 de mayo de 2009. Disponible en: http://www.inspection.gc.ca/ english/fssa/concen/cause/listeriae.shtml.

9. Evans MR, Swaminathan B, Graves LM, Altremann E, Klaenhammer TR, Fink RC, et al. Genetic markers unique to Listeria monocytogenes serotype $4 \mathrm{~b}$ differentiate epidemic clone II (hot dog outbreak strains) from other linages. Appl Environ Microbiol. 2004;70:2383-90.

10. Gilbreth E, Call JE, Wallace M, Scott VN, Chen Y, Luchansky JB. Relatedness of Listeria monocytogenes isolated recovered from selected ready to eat foods and listeriosis patients in the United States. Appl Environ Microbiol. 2005;17:8115-22.

11. Volpe KE, Kathariou S, Edwards JS, Wolf LA. Multiplelocus variable-number tadem-repeat analysis as a tool for subtyping Listeria monocytogenes strain. J Clin Microbiol. 2008;46:1435-50.

12. Oteo J, Alós Jl. Listeria y listeriosis. Fecha de consulta: 31 de mayo de 2009. Disponible en: http://www.seimc.org/ control/revisiones/bacteriologia/listeria.pdf.

13. Mammina C, Aleo A, Romani C, Pellissier N, Nicoletti $P$, Pecile $\mathbf{P}$, et al. Characterization of Listeria monocytogenes isolates from human listeriosis cases in Italy. J Clin Microbiol. 2009;47:2925-30.

14. Wikipedia. Listeriosis. Fecha de consulta: 31 de mayo de 2009. Disponible en: http://es.wikipedia.org/wiki/Listeriosis.

15. Food Drug Administration. Foodborne pathogenic microorganisms and natural toxins handbook. Listeria monocytogenes. Fecha de consulta: 31 de mayo de 2009. Disponible en:_http://www. fda.gov/Food/FoodSafety/Foodbornelllness/ FoodbornelllnessFoodbornePathogensNaturalToxins/ BadBugBook/ucm070064.htm.

16. U.S. Food and Drug Administration. Listeria monocytogenes: Draft guidance. Sec. 555.320.
Washington, D.C.: FDA; 2008. p. 7. Fecha de consulta: 31 de mayo de 2009. Disponible en: http://www.fda.gov/ICECI/ ComplianceManuals/CompliancePolicyGuidanceManual/ ucm136694.htm.

17. U.S. Food and Drug Administration. Guidance for industry: Control of Listeria monocytogenes in refrigerated or frozen ready-to-eat foods; draf guidance. Washington: FDA; 2008. p. 26. Fecha de consulta: 31 de mayo de 2009. Disponible en: http://www.fda. gov/food/guidancecomplianceregulatoryinformation/ guidancedocuments/foodprocessinghaccp/ucm073110. $\mathrm{htm}$.

18. U.S. Food and Drug Administration. Listeria: Washington: FDA, 2008. p. 3. Fecha de consulta: 31 de mayo de 2009. Disponible en: http://www.fda.gov/ForConsumers/ ByAudience/ForWomen/ucm118542.htm.

19. Medline Plus. Listeriosis: infecciones por Listeria. Biblioteca Nacional de Medicina de EE.UU. 2010. Fecha de consulta: 31 de mayo de 2010. Disponible en: http://www.nlm.nih.gov/ medlineplus/spanish/listeriainfections.html.

20. Kim JW, Siletzky RM, Kathariou S. Hot ranges of Listeria -specific bacteriophages from the turkey processing plant environment in the United States. Appl Environ Microbiol. 2008;74:6623-30.

21. Organización Mundial de Sanidad Animal. Manual de la Organización Mundial de Sanidad Animal (OIE) sobre animales terrestres. Cap. 210.14. Listeria monocytogenes. Madrid: OIE; 2004. p. 16.

22. Perroni MA. Listeria monocytogenes y su presencia en productos lácteos. Diaeta. 2008;26:37-43.

23. Centers for Disease Control and Prevention; National Center for Zoonotic, Vector-Borne, and Enteric Diseases. Listeriosis. Atlanta: CDC. (Your Online Source for credible). Fecha de consulta: 31 de mayo de 2009. Disponible en: http://www.cdc.gov/nczved/divisions/dfbmd/ diseases/listeriosis/.

24. Stelten A, Nightingale KK. Development and implementation of a multiplex single -nucleotide polymorphism genotyping assay for detection of virulence attenuating mutations in the Listeria monocytogenes virulence-associated gene InIA. Appl Environ Microbiol. 2008;74:7365-75.

25. United States Department of Agriculture. Food Safety and Inspection Service. Protec your baby and yourself from listeriosis. USA: FSIS; 2006. Fecha de consulta: 31 de mayo de 2009. Disponible en: http://www.fsis.usda.gov/ factsheets/Protect_Your_Baby/index.asp.

26. Álvarez CJ, Guerrero JA, Muñoz MN, López MP, Borbón ME, Espinosa J. Informe epidemiológico de la vigilancia de eventos grupo factores de riesgo ambiental-Colombia 2010. Bogotá: INS; 2010. p. 25.

27. Jay JM. Microbiología moderna de los alimentos. En: Listeriosis transmitidas por alimentos. Tercera edición. Zaragoza (España): Editorial Acribia, S.A., 1994. p. 601-49.

28. Food Safety Network. Listeria monocytogenes: What is L. monocytogenes? Canada: FSN, 2010. (FSN Food Riskes). Fecha de consulta: 30 de mayo de 2010. Disponible en: http://www.foodsafetynetwork.ca/aspx/ public/publication_detail_global.aspx?languageid=1\&con tenttypeid $=5 \& i d=76$. 
29. Walker SJ, Archer P, Banks JG. Growth of Listeria monocytogenes at refrigeration temperatures. J Appl Bact. 1990;68:157-62.

30. Pan Y, Breidt F Jr, Kathariou S. Competition of Listeria monocytogenes serotype $1 / 2$ a and $4 \mathrm{~b}$ strains in mixedculture biofilms. Appl Environ Microbiol. 2009;75:5846-52.

31. Shen Y, Liu Y, Zhang Y, Cripe J, Conway W, Meng J, et al. Isolation and characterization of Listeria monocytogenes isolates from ready to eat foods in Florida. Appl Environ Microbiol. 2006;72:5073-6.

32. Gilmour MW, Graham M, Domselaar GV, Tyler S, Kent H, Trout-Yake KM, et al. High-throughput genome sequencing of two Listeria monocytogenes clinical isolates during a large foodborne outbreak. BMC Genomics. 2010;11:1471-85.

33. Cole NB, Jones MV, Holyoak C. The effect of $\mathrm{pH}$, salt concentration and temperature on the survival and growth of Listeria monocytogenes. J Appl Bacterio. 1990;69:63-72.

34. Liu D, Lawrence LM, Gorski L, Mandrell RE, Ainsworth AJ, Austin FW. Listeria monocytogenes serotype 4b strains belonging to lineages I and III possess distinct molecular features. J Clin Microbiol. 2006;44:214-7.

35. Wong AC. Biofilms in food processing environments. J Dairy Sci. 1998;81:2765-70.

36. Nightingale KK, Windham K, Wiedmann M. Evolution and molecular phylogeny of Listeria monocytogenes isolated from human and animal listeriosis cases and foods. J Bacteriol. 2005;187:5537-51.

37. Berche P, Gaillard JL, Richard S. Invasiveness and intracellular growth of Listeria monocytogenes. Infection. 1988;16(Suppl.2):145-8.

38. Sánchez E, Pardo R. Listeriosis del sistema nervioso central, formas meníngeas agudas supurativas y romboencefálica. Acta Neurol Colomb. 1992;8:165-8.

39. Brun- Buisson CJ, de Gialluly E, Gherardi R, Otterbein G, Gray F, Rapin M. Fatal nonmeningitic Listeria rhomboencephalitis, report of two cases. Arch Int Med. 1985;145:1982-5.

40. Armstron D. Listeria monocytogenes. En: Mandel GL, Douglas RG, Benett JE, editors. Principles and practice of infectious diseases. $4^{\text {th }}$ edition. New York: Churchill Livingtone; 1995. p. 1180-5.

41. Payán A, Estudillo M. Listeriosis neonatal: ¿enfermedad poco frecuente o no diagnosticada? Enfoque microbiológico. Colombia Médica.1994;25:69-72.

42. Sánchez CE, Castillo A, Ortega C. Infección perinatal por Listeria. Reporte de un caso. Rev Col Obstet Ginecol. 2001;52:190-202.

43. Dalton CB, Austin CC, Sobel J, Hayes PS, Bibb WF, Graves LM, et al. An outbreak of gastroenteritis and fever due to Listeria monocytogenes in milk. N Engl J Med. 1997;336:100-5.

44. Salamina G, Dalle DE, Niccolini A, Poda G, Cesaroni D, Bucci M, et al. A foodborne outbreak of gastroenteritis involving Listeria monocytogenes. Epidemiol Infect. 1996;117:429-36.

45. Dalton CB, Med B, Austin CC, Sobel J, Hayes PS, Bibb WF, et al. An outbreak of gastroenteritis and fever due to Listeria monocytogenes in milk. N Engl J Med.1997;336:100-5.
46. United States Department of Agriculture. Food Safety and Inspection Service. At- risk populations. USA: FSIS; 2010. Fecha de consulta: 28 de mayo de 2010. Disponible en: http:// ucfoodsafety.ucdavis.edu/Consumer_Advice/Foodborne_ Illness_-_At_Risk_Populations/.

47. Kagkli DM, Iliopoulos V, Stergiou V, Lazaridou A, Nychas GJ. Differential Listeria monocytogenes strain survival and growth in katiti, a traditional Greek soft cheese, at different storage temperaturas. Appl Environ Microbiol. 2009;75:3621-6.

48. Borucki MK, Kim SH, Call DR, Smole SC, Pagotto F. Selective discrimination of Listeria monocytogenes epidemic strains by a mixed-genome DNA microarray compared to discrimination by pulse-field gel electrophoresis, ribotyping, and multilocus sequence typing. J Clin Microbiol. 2004;42:5270-6

49. Nelson KE, Fouts DE, Mongodín EF, Ravel J, Deboy $\mathbf{R}$, Kolonay JF, et al. Whole genome comparisons of serotype $4 b$ and $1 / 2$ a strains of the food-borne pothogen Listeria monocytogenes reveal new insights into the core genome components of this species. Nucleic Acids Res. 2004;32:2386-95.

50. Bruhn JB, Vogel BF, Gram L. Bias in the Listeria monocytogenes enrichment procedure: Linaje 2 strains out compete linaje 1 strains in University of Vermont selective enrichments. Appl Environ Microbiol. 2005;71:961-7.

51. Kathariou S. Listeria monocytogenes virulence and pathogenicity, a food safety perspective. J Food Prot. 2002;65:1811-29.

52. Wikipedia. Brote de listeriosis de 2008-2009 en Chile. Chile: Ministerio de Salud, 2010. Fecha de consulta: 29 de mayo de 2010. Disponible en: http://es.wikipedia.org/wiki/ brote_de_listeriosis_de_2008-2009_en Chile.

53. Linnan MJ, Mascola L, Lou XD, Goulet V, May S, Salminen C, et al. Epidemic listeriosis associated with Mexican -style cheese. N Engl J Med. 1988;319;823-8.

54. Centers for Disease Control and Prevention. Outbreak of listeriosis associated with homemade Mexican-Style Cheese, North Carolina, October 2000, and January 2001. MMWR Morb Mortal Wkly Rep. 2001;50:560-2.

55. Fleming DW, Cochi SL, MacDonald KL, Brondum J, Hayes PS, Plikaytis BD, et al. Pasteurized milk as a vehicle of infection in an outbreak of listeriosis. N Engl Med. 1985;312:404-7

56. McLauchlin J, Hall SM, Velani SK, Gilbert RJ. Human listeriosis and pate-a possible association. Brit Med J.1991;303:773-5.

57. Rocourt J. Listeria monocytogenes: The state of the science. Dairy Food Environ San. 1994;14:70-82.

58. Callejo R, Prieto M, Martínez C, Aguerre L, Rocco F, Martínez G. Manual de procedimientos: aislamiento, identificación, y caracterización de Listeria monocytogenes. Buenos Aires, Argentina: WHO Global Salm Surv; 2008. p. 39.

59. Schlech WF, Lavigne PM, Bortolussi RA, Allen AC, Haldane EV, Wort AJ, et al. Epidemic listeriosis-evidence for transmission by food. N Engl J Med. 1983;308:203-6.

60. Kerr KG, Dealler SF, Lancey RW. Materno fetal listeriosis from cook-chill and refrigerated food. Lancet. 1988;2:1113. 
61. Shwartz B, Hexter D, Broome CV, Higtower AW, Hirschhorn RB, Porter JD. Investigation of an outbreak of listeriosis: New hypothesis for the etiology of epidemic Listeria monocytogenes infection. J Infect Dis. 1989;159:680-5.

62. Goulet V, Lepautre A, Rocourt J. Epidémie de listériose en France-Bilan final et resultats de l'enquête épidémiologique. Bull Epid Hebdom. 1993;4:13-4.

63. Wulff G, Gram L, Ahrens P, Vogel BF. One group of genetically similar Listeria monocytogenes strains frequently dominates and persists in several fish slaughter- and smokehouses. Appl Environ Microbiol. 2006;72:4313-22.

64. Food Safety and Inspection Service .U.S. Department of Agriculture. Microbiological testing programs for ready-toeat (RTE) meat and poultry products. Washington: FSIS, 2005.

65. Yildirim S, Lin W, Hitchen AD, Jaykus LA, Alterman E, Klaenhammer TR, et al. Epidemic clone 1-specific genetic markers in strains of Listeria monocytogenes serotype 4b from foods. Appl Environ Microbiol. 2004;70:4158-64.

66. Cruz CD, Silvestre FA, Kinoshita EM, Landgraf M, Franco BDGM, Destro MT. Epidemiological survey of Listeria monocytogenes in a gravlas salmon processing line. Braz J Microbiol. 2008;39:375-83.

67. United States Department of Agriculture. Percent positive Listeria monocytogenes test for RTE meat and poultry, CY 2008. USA: FSIS; 2009.

68. Najjar MB, Chikindas M, Montville T. Changes in Listeria monocytogenes membrane fluidity in response to temperatures stress. Appl Environ Microbiol. 2007;73:642935

69. Yoon Y, Kendall PA, Belk KE, Scanga JA, Smith GC, Sofos JN. Modeling the grow/no-growth boundaries of postprocessing Listeria monocytogenes contamination on frankfurters and Bologna treated with latica cid. Appl Environ Microbiol. 2009;75:353-8.
70. Food and Drug Administration. Bacteriologycal analytical manual online. Detection and enumeration of Listeria monocytogenes in foods. U.S.A: FDA, 2003. Fecha de consulta: 27 de mayo de 2009. Disponible en: http:// www.fda.gov/food/scienceresearch/laboratorymethods/ bacteriologicalanalyticalmanualbam/ucm071400.htm.

71. Food Drug and Administration. Método adaptado para detección de Listeria monocytogenes en carnes y derivados cárnicos, huevos, pollos: metodología de la Food Drug Administration para la detección de Listeria monocytogenes en alimentos. USA: BAM; 2003. p. 30.

72. Food and Agriculture Organization of the United Nations. World Health Organization. Codex Alimentarius commission. Annex II: Microbiological criterio for Listeria monocytogenes in ready-to-eat food. (CX /FH 08/40/5, October 2008). Codex Committee on Food Hygiene, fortieth session. Guatemala: FAO/WHO; 2008. p. 8.

73. Seeliger HP, Hönne K. Serotyping of Listeria monocytogenes and related species. Vol.13. In: Bergan T, Novis J, eds. Methods in microbiology. Capítulo 11. London, New York: Academic Press, 1979. p.31-49

74. Institut Pasteur. Centre National de Référence des Listeria. Centre collaborateur de L'oms pour la listeriose d'origene alimentaire. Laboratoire des Listeria. Protocole de serotypie. Paris: Institut Pasteur, 1997 : 22p.

75. Departamento Administrativo Nacional de Estadística. Necesidades básicas insatisfechas -NBI. Bogotá: Dane, 2008. Fecha de consulta: 10 de junio de 2010. Disponible en: http: // www.dane.gov.co/daneweb_VO9/index.php.

76. República de Colombia. Departamento Nacional de Planeación. Consejo Nacional de Política Económica y Salud. Documento Conpes 3375. Política nacional de sanidad agropecuaria e inocuidad de alimentos para el sistema de medidas sanitarias y fitosanitarias. Bogotá D. C: DNP, 2005. 39p. 